\title{
Simulating Distributed and Global Consciousness Under Spatial Grasp Paradigm
}

\author{
Peter Simon Sapaty
}

Institute of Mathematical Machines and Systems National Academy of Sciences, Glushkova Ave 42, 03187 Kiev Ukraine

\author{
*Corresponding author \\ Peter Simon Sapaty, Institute of Mathematical Machines and Systems \\ National Academy of Sciences, Glushkova Ave 42, 03187 Kiev Ukraine
}

Submitted: 23 Nov 2020; Accepted: 02 Dec 2020; Published: 12 Dec 2020

\begin{abstract}
The paper is investigating the possibility of using developed and tested in different countries Spatial Grasp model and technology (SGT) for simulating global awareness and consciousness in distributed dynamic systems, with potential applications in intelligent system management, industrial development, space research, security, and defense. The main technology component, Spatial Grasp Language (SGL), allows us to obtain powerful and compact spatial solutions of different problems by directly expressing their top semantics while hiding traditional system organization and management routines inside efficient networked implementation. The paper describes in SGL a traditional organization of two opposing swarms, called "chasers" and "targets", randomly operating on expected area. It then enriches the chasers swarm with global awareness and a sort of migrating consciousness, further strengthened by external super-consciousness capability, which allows it to drastically improve performance and make important nonlocal decisions, while putting it to a superior position over the opposing targets swarm. Despite simplicity of the shown practical example, it gives hope for the use of SGT for simulation of much broader and complex areas linked with consciousness like, for example, brain's bimolecular processes and the basic structure of the universe. The developed networking technology can be readily implemented even in traditional university environments, as was done in the past for its previous versions in different countries under the author's supervision.
\end{abstract}

Keywords: Global awareness, Migrating consciousness, External consciousness, Global consciousness, Spatial Grasp Technology, Spatial Grasp Language, Parallel distributed simulation, Collective behavior and Swarming, Structure of the universe.

\section{Introduction}

This paper relates to such mysterious topic and problem as consciousness, with nobody knowing what it actually means, only having intuitive feelings of its existence and numerous guesses of what it might be. These feelings, for example, include that consciousness is everything you experience and consists of whatever you happen to be aware of at any given moment in time, also reflecting perceptions of the outside world, and so on. Many consider it like mechanism by which it occurs in the brain, others think that its ultimate place in the universe is unknown. There exists a great deal of literature on very different, often fantastic and futuristic, ideas about the nature and actual locations of consciousness, like does it relate to human beings only, can exist in higher level animals too, can also be found in any matter like stones, water, clouds, and so on. Or it pervades the creation of the universe and is brought to the brain from outside, also leaving it and continuing to exist after people die.

Here we can only name some directions of the research in the consciousness area, with references to related sources, which may include the following: what is consciousness, artificial consciousness, spatial consciousness, distributed consciousness, global consciousness, social consciousness, stream of consciousness, visual perception and consciousness, consciousness outside of the brain, consciousness pervades the universe, consciousness in the universe, network theory and consciousness, the qualities of consciousness, and very many others [1-13]. Among the studied qualities of consciousness, the following can be named: all possibilities, harmonizing, freedom, unboundedness, infinite dynamism, self-sufficiency, integration, infinite organizing power, invincibility, perfect orderliness, perfect balance, fully awake within itself, evolutionary, simplicity, immortality, and others [13].

The paper investigates how to use the developed Spatial Grasp paradigm and Technology (SGT) for simulation of global awareness and consciousness in distributed intelligent systems. SGT, patented and revealed in numerous publications (Wiley, Springer, and Emerald books including), provides basics for deep integration, actually symbiosis, of different worlds by allowing us to unite advanced distributed simulation with spatial parallel and fully distributed control [14-21]. The investigated applications included classical graph and network theory problems, missile defense, massive collective robotics, evolution of space systems, flexible command and control, industrial, social and international security problems, also expressed main gestalt theory laws allowing 
them to cover any distributed systems rather than just human mind and brain. The developed formalism allows us to directly exist, operate, and move in different worlds and their combinations, while shifting traditional numerous and boring system management and simulation routines to automatic networked interpretation of the basic Spatial Grasp Language (SGL), with the resulting solutions often hundreds of times shorter and simpler than usual.

The rest of the paper is as follows. Section 2 describes SGT basics, including SGL and its networked interpreter. Section 3 provides expression in SGL of two opposing swarms called "chasers" and "targets", randomly propagating and covering certain operational region and capable of fighting each other. Section 4 provides a snapshot in SGL for global awareness for the chaser's swarm over the whole operational area and all units there. Section 5 implants this global awareness into a sort of constantly active and spatially migrating consciousness for the chaser's swarm, allowing the latter to improve performance, analyze nonlocal situations in the operational area, and make effective decisions, giving it big advantage over the opposing targets swarm. Section 6 shows how to organize higher level or super-consciousness for the chaser's swarm, by continually analyzing from outside the presence of migrating consciousness in it, with re-launching this wandering consciousness if accidentally terminated by the failure of some chaser's units. Section 7 concludes the paper with disclosing plans on the further research in this area and possibilities of quick reimplementation of SGT even in traditional university environments, as was done earlier in different countries.

\section{Spatial Grasp Technology Basics}

\section{General SGT Idea}

Within Spatial Grasp Technology (SGT), a high-level scenario for any task to be performed in a distributed world is represented as an active self-evolving pattern rather than traditional program, sequential or parallel. This pattern, written in a high-level Spatial Grasp Language (SGL) and expressing top semantics of the problem to be solved, can start from any world point. It then spatially propagates, replicates, modifies, covers and matches the distributed world in parallel wavelike mode, while echoing the reached control states and data found or obtained for making decisions at higher levels and further space navigation. This inherently parallel and fully distributed spatial process is symbolically shown in (Figure $1 \mathrm{a}, \mathrm{b}$ ) where reached physical or virtual world points, whatever remote they happen to be, can launch new spatial wave processes remaining under control from the previous points or becoming independent from them.
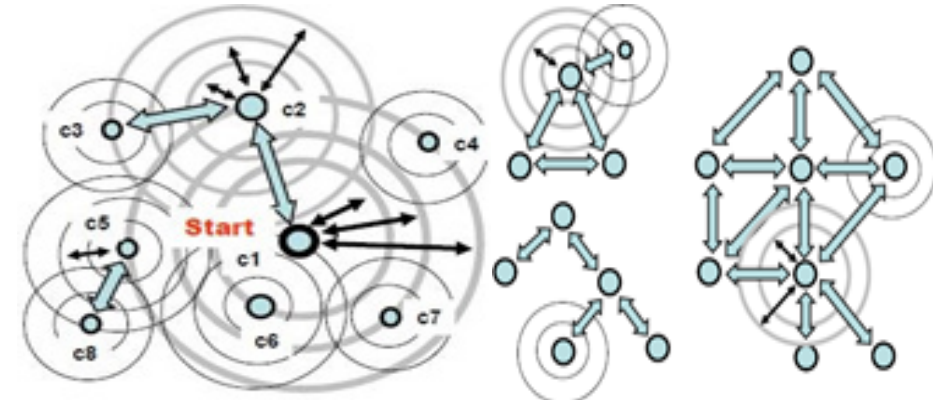

Figure 1: Controlled navigation \& coverage \& matching \& grasping of distributed spaces
Many spatial processes in SGL can start any time and in any places, cooperating or competing with each other, depending on applications. The self-spreading \& self-matching SGL patternsscenarios can create knowledge infrastructures arbitrarily distributed between system components, which may cover any regions, from terrestrial (Figure $2 \mathrm{a}$ ) to even celestial in the future (Figure 2b).

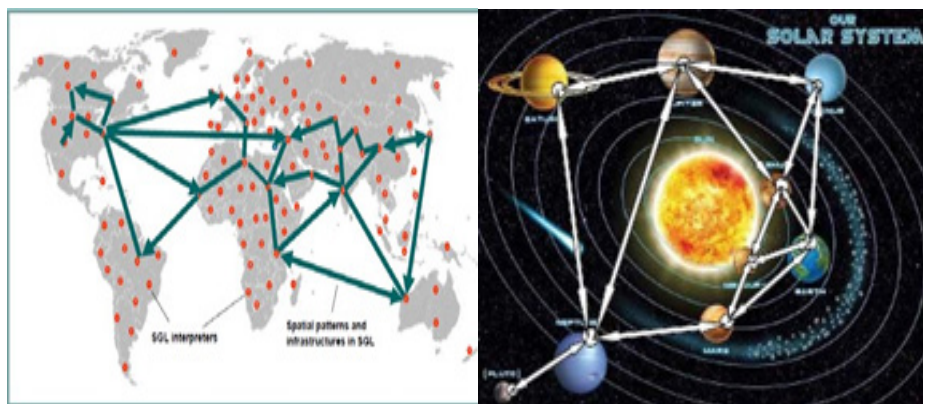

Figure 2: Spreading spatial patterns and creation of distributed infrastructures

These temporary or persistent spatial infrastructures, which may remain active and launch new spatial waves anytime (as in Figure 1b), can effectively support distributed databases, advanced command and control, situation awareness, autonomous and collective decisions. They can also express any existing or hypothetical biological, computational and world creation models (say, related to neural nets, Petri nets, quantum biology, neuroscience, physics and cosmology).

\section{Spatial Grasp Language}

General SGL organization is as follows, where syntactic categories are shown in italics, vertical bar separates alternatives, parts in braces indicate zero or more repetitions with a delimiter at the right if multiple, and constructs in brackets may be optional:

grasp $\rightarrow$ constant $\mid$ variable $\mid[$ rule $][(\{$ grasp,$\})]$

From this definition, an SGL scenario called grasp, supposedly applied in some point of the distributed space, can just be a constant directly providing the result to be associated with this point. It can be a variable whose content, assigned to it previously when staying in this or other space point (as variables may have nonlocal meaning and coverage), provides the result in the application point too. It can also be a rule (expressing certain action, control, description or context) optionally accompanied with operands separated by comma (if multiple) and embraced in parentheses. These operands can be of any nature and complexity (including arbitrary scenarios themselves) and defined recursively as grasp too, i.e. can be constants, variables or any rules with operands (i.e. as grasps again), and so on.

Rules, starting in some world point, can organize navigation of the world sequentially, in parallel or any combinations thereof. They can result in staying in the same application point or can cause movement to other world points, with obtained results to be left there, as in the rule's final points. Such results can also be collected, processed, and returned to the rule's starting point, the latter serving as the final one on this rule. The final world points reached after the rule invocation can themselves become starting ones for 
other rules. The rules, due to recursive language organization, can form arbitrary operational and control infrastructures expressing any sequential, parallel, hierarchical, centralized, localized, mixed and up to fully decentralized and distributed algorithms. These algorithms, called spatial, can effectively operate in, with, under, in between, over, and instead of (as for simulation) large, dynamic, and heterogeneous spaces, which can be physical, virtual, management, command and control, or combined.

SGL full syntax description, as of its latest version, is as follows, with the words in Courier New font being direct language symbols (boldfaced braces including).

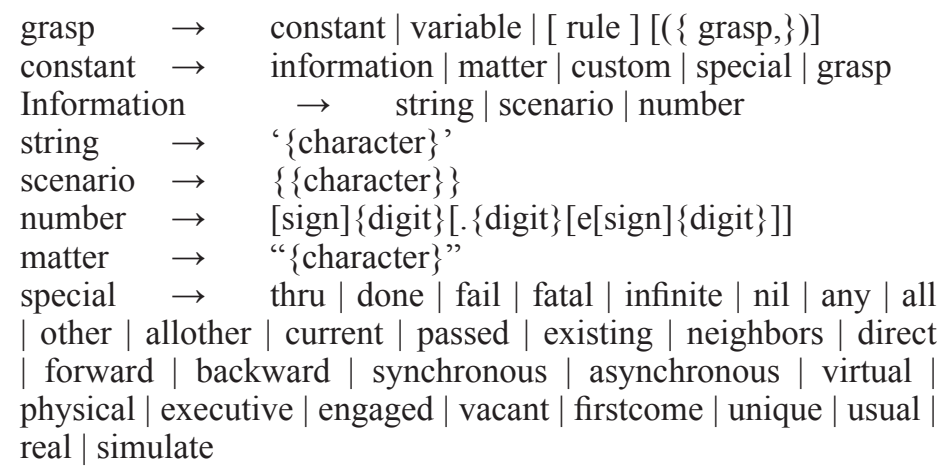

variable $\rightarrow$ global | heritable $\mid$ frontal | nodal | environmental

global $\rightarrow$ G $\{$ alphameric $\}$

heritable $\rightarrow$ H alphameric

frontal $\rightarrow$ F \{alphameric $\}$

nodal $\rightarrow \quad \mathrm{N}$ alphameric

environmental $\rightarrow$ TYPE | IDENTITY | NAME

CONTENT | ADDRESS | POINT | QUALITIES | WHERE BACK | PREVIOUS | PREDECESSOR | DOER | RESOURCES | LINK |

rule $\rightarrow$ DIRECTION | WHEN | TIME | STATE | VALUE IDENTITY | IN | OUT | STATUS | MODE | COLOR

type | usage | movement | creation | echoing | verification assignment $\mid$ advancement $\mid$ branching $\mid$ transference $\mid$ exchange $\mid$ timing | qualifying | grasp

type $\quad \rightarrow \quad$ global $\mid$ heritable $\mid$ frontal $\mid$ nodal $\mid$ environmental |matter | number | string | scenario | constant | custom

usage $\rightarrow$ address $\mid$ coordinate $\mid$ content $\mid$ index $\mid$ time $\mid$ speed $\mid$ name | place $\mid$ center $\mid$ range $\mid$ doer $\mid$ node | link | unit

movement $\rightarrow$ hop | hopfirst | hopforth | move | shift | pass | return $\mid$ follow

creation $\rightarrow \quad$ create $\mid$ form $\mid$ linkup | delete | unlink

echoing $\rightarrow \quad$ state $\mid$ rake $\mid$ order $\mid$ unit $\mid$ unique $\mid$ sum $\mid$ count $\mid$ first $\mid$ last $|\min | \max \mid$ random $\mid$ average $\mid$ sortup | sortdown | reverse | element $\mid$ position $\mid$ fromto $\mid$ add $\mid$ subtract $\mid$ multiply | divide degree $\mid$ separate $\mid$ unite $\mid$ attach $\mid$ append $\mid$ common | withdraw increment $\mid$ decrement $\mid$ access $\mid$ invert $\mid$ apply | location verification $\rightarrow$ equal $\mid$ nonequal | less | lessorequal | more | moreorequal | bigger | smaller | heavier | lighter | longer | shorter | empty | nonempty | belong | notbelong | intersect | notintersect | yes $\mid$ no

assignment $\quad \rightarrow \quad$ assign $\mid$ assignpeers $\mid$ associate

advancement $\rightarrow$ advance $\mid$ slide $\mid$ repeat $\mid$ align $\mid$ fringe

branching $\quad \rightarrow \quad$ branch $\mid$ sequence $\mid$ parallel $\mid$ if $\mid$ or $\mid$ and | choose | quickest | cycle |loop | sling | whirl | split

transference $\quad \rightarrow \quad$ run $\mid$ call

exchange $\quad \rightarrow \quad$ input $\mid$ output $\mid$ send $\mid$ receive $\mid$ emit $\mid$ get

timing $\quad \rightarrow \quad$ sleep $\mid$ allowed

qualification $\rightarrow$ contain $\mid$ release $\mid$ free $\mid$ blind $\mid$ quit $\mid$ abort | stay | lift | seize

\section{SGL Interpreter}

The SGL interpreter main components and its general organization are shown in Figure 3.

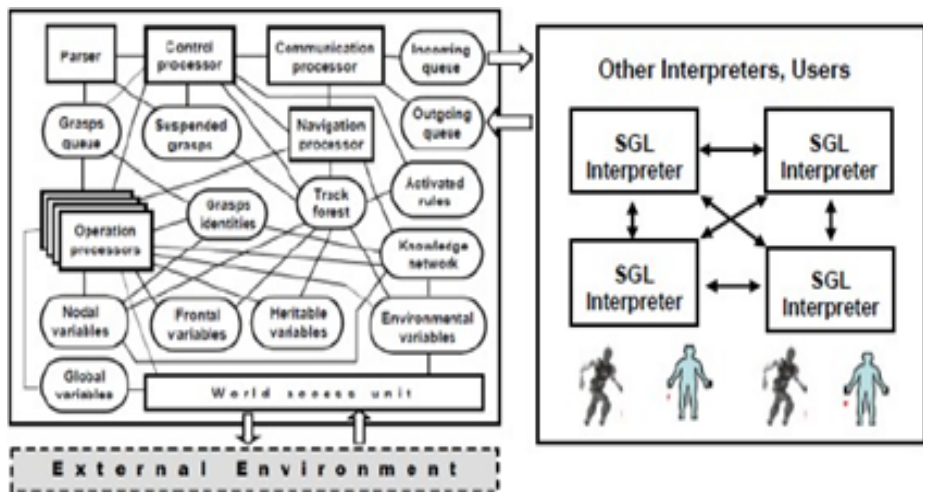

Figure 3: SGL interpreter main components and their interactions, also links with the external world

The interpreter consists of a number of specialized functional processors (shown by rectangles) working with and sharing specific data structures. These include: Communication Processor, Control Processor, Navigation Processor,

Parser, different Operation Processors, and special (external \& internal) World Access Unit directly manageable from SGL. Main data structures (also referred to as stores) with which these processors operate (shown by ovals) comprise: Grasps Queue, Suspended Grasps, Track Forest, Activated Rules, Knowledge Network, Grasps Identities, Heritable Variables, Fontal Variables, Nodal Variables, Environmental Variables, Global Variables, Incoming Queue, and Outgoing Queue. SGL interpretation network, which can comprise any number of communicating interpreters distributed terrestrially or even celestially, generally serves multiple scenarios or their parallel branches simultaneously navigating the distributed world, which can cooperate or compete with each other. 
As both backbone and nerve system of the distributed interpreter, its hierarchical spatial track system dynamically spans the worlds in which SGL scenarios evolve, providing automatic control and management of multiple distributed processes. Its part related to the current interpreter is kept in the Track Forest store (see Figure 3 ) which is interlinked with similar parts in other interpreters, forming altogether the global control coverage. Self-optimizing in parallel echo processes, this (generally forest-like) distributed track structure provides hierarchical command and control as well as remote data and code access. It also supports spatial variables and merges distributed control states for making decisions at different organizational levels. The track infrastructure can be automatically distributed between different world points during scenario spreading in distributed environments.

Each interpreter can support and process multiple SGL scenario code which happens to be in its responsibility at different moments of time. More details on SGT, SGL, its implementation and investigated and tested applications can be found elsewhere, including in [14-19]. Implanted into any distributed systems and integrated with them, the interpretation network (having potentially millions to billions of communicating interpreter copies) allows us to form a spatial world computer with practically unlimited power for simulation and management of the whole world.

Collective Randomized Movement of Two Opposing Swarms We will consider here the situation where one swarm of units named "chasers" and another one called "targets" can randomly propagate and interact with each other on some initially expected scenario area, as in Figure 4.

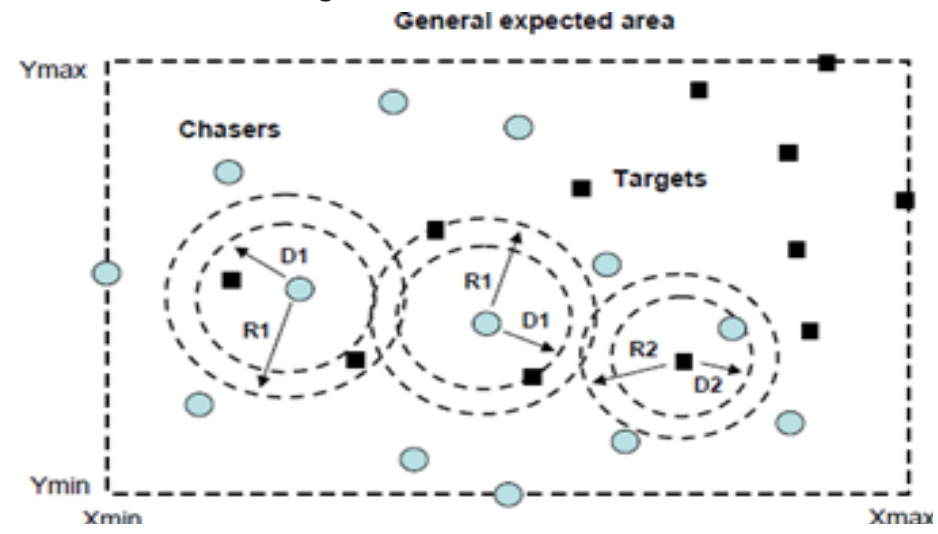

Figure 4: Two opposing swarms operating and interacting on some expected area

During their collective propagation, individual chasers can destroy targets seen in certain distance (up to D1), with targets also capable of fatally damaging chasers if appear within certain reach (up to D2). The randomized movement of chasers and targets should be organized in such a way that units of the same type have to keep some threshold distance from each other (not closer than by radius R1 for chasers, and radius R2 for targets), for efficient coverage of the whole region. It is supposed that the main functionality of targets is to collect and accumulate certain intelligence in the points occupied, and the main task of chasers is to neutralize targets discovered, as much as they can. Targets can also attack chasers if too close to them, to protect themselves, but with presumably lesser power than that of chasers. That means that both chasers and targets can gradually reduce in their numbers during the simulation period.

The SGL scenarios for swarming chasers (with names C1, C2, ..., Cm) and targets (called T1, T2, .., Tn) will be as follows, where all units in these swarms are initially accessed and tasked in parallel from some external position.

\section{- Chasers swarming}

hop nodes $(\mathrm{C} 1, \mathrm{C} 2, \ldots . \mathrm{Cm})$;

nodal $($ Area $=($ Xmin, Xmax, Ymin, Ymax $), \mathrm{R} 1=\ldots, \mathrm{D} 1=\ldots)$; move_random(Area));

repeat

Shift $=\operatorname{random}(\mathrm{dx}$ dy $) ;$ New $=$ WHERE + Shift;

if ((inside (New, Area); notseen (New, R1, chasers)), shift(Shift)); if (detect (targets, D1), select_destroy(targets));

sleep(delay))

\section{- Targets swarming}

hop_nodes $(\mathrm{T} 1, \mathrm{~T} 2, \ldots, \mathrm{Tn})$;

nodal $($ Area $=($ Xmin, Xmax, Ymin, Ymax $), \mathrm{R} 2=\ldots, \mathrm{D} 2=\ldots)$; move(random(Area));

repeat

Shift $=$ random $\left(\mathrm{dx} \_\right.$dy $) ;$New $=$WHERE + Shift;

if ((inside (New, Area); notseen (New, R2, targets)), shift(Shift)); collect (WHERE, Intelligence);

if (detect (chasers, D2), select_attack(chasers)); sleep(delay))

\section{Providing Global Awareness to the Swarm Operation}

In the previous SGL scenarios, the chaser and target units were operating in a fully distributed way, with keeping certain distances from each other while always remaining within the planned operational area, also making individual local decisions whether to move further and interact with other units, which may be considered as traditional for swarming organizations. By enriching a certain swarm with a sort of global awareness over the operational area, and using this awareness to influence local decisions and actions, may essentially improve its performance locally and as a whole, and even guarantee survivability in complex situations.

For the chasers, such global awareness may, for example, include knowledge of the total number of chasers in their swarm, which may be obtained by contacting from any chaser all other chasers, itself including (directly or stepwise in a spanning tree mode via direct communications between them, depending on SGL implementation), as in Fig. 5. Other knowledge of interest may concern the existing targets, say, by using a sort of radar capabilities for remote objects detection (which some or all chasers may possess), including their full number and coordinate dimensions of the total area occupied by them, see Fig. 5 too, and also the total number of targets destroyed by all chasers. And all these parameters may be quite different in different moments of observation time as the swarms of both types are on constant move with capability of attacking and destroying each other. 


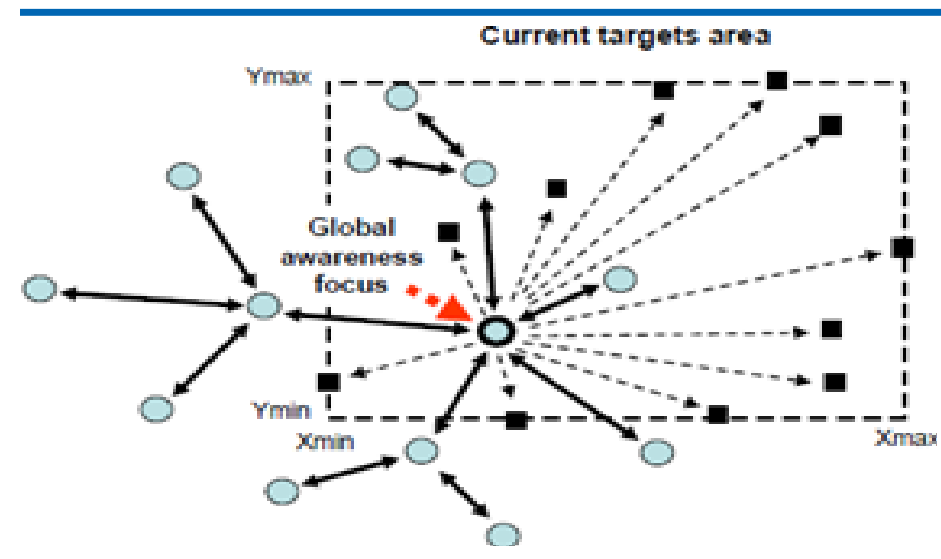

Figure 5: Collecting global information on the moving swarms

The SGL description of obtaining a snapshot of such global awareness in some moment of time may be as follows.

nodal (Chasers_number, Targest_number, Targets_area, Targets destroyed); hop_random(chasers);

Chasers_number $=$ count $($ hop(all_chasers));

Targets_number $=$ count(hop(all_chasers); radar(targets)); Targets_area = boundaries(hop(all_chasers); $\operatorname{radar}(\operatorname{targets})$ ); Targets_destroyed =sum(hop(all_chasers); Killed)

\section{Migrating Consciousness Based on Global Awareness}

To convert a global snapshot of the area and units inhabiting it, described in the previous section, into a constantly working global awareness up to a sort of consciousness regularly delivered to all chasers and influencing their functionality, also getting feedback from the chasers on the number of targets they have destroyed, we may extend the previous SGL scenario as follows. This will also organize the focuses of such consciousness as constantly migrating between the chasers, as in Fig. 6, in order to minimize danger of being destroyed by targets, with the proper use of frontal and nodal variables for transferring data to and from the individual chasers, also preserving full functionality of such spatially wandering consciousness.

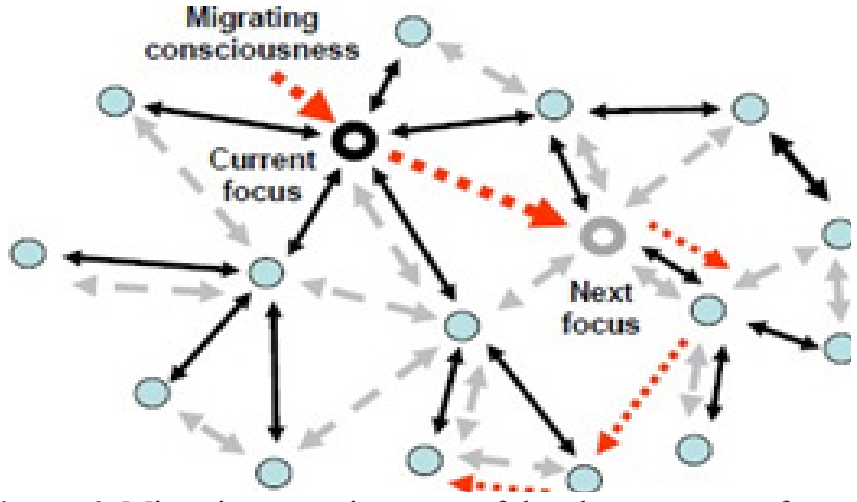

Figure 6: Migrating consciousness of the chasers swarm frontal

Chases_number, Targest_number, Targets_area, Targets destroyed); Nodal (C_number, T_number, Area, T_destroyed); repeat (hop_random(chasers); stay $($ Chasers_number $=\operatorname{count}($ hop(all_chasers $))$;

Targets_number $=$ count(hop(all_chasers); $\operatorname{radar}(\operatorname{targets}))$; Targets_area = boundaries(hop(all_chasers); $\operatorname{radar}(\operatorname{targets}))$; hop(all_chasers);

C_number $=$ Chasers_number;

T_number $=$ Targets_number;

Area $=$ Targets_area;

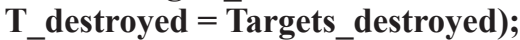

sleep(delay))

In the above scenario, there is a regular exchange between frontal variables (like Chases_number, Targets_number, Targets_area, and Targets_destroyed) serving and supporting the wandering consciousness process, and the equivalent nodal variables (like $\mathrm{C}_{-}$ number, $\mathrm{T}$ number, Area, and $\mathrm{T}$ destroyed) in each chasers unit. Where the Targets area discovered and collected by the global awareness substitutes the original Area information in the chasers (supposed common for chasers and targets at the beginning), which are now becoming oriented only on operating within the area where all targets are observed, with immediate moving into this area (which itself may constantly change), if happen to be outside of it. The above migrating consciousness scenario highlights in bold its interaction part with the updated functionally of chasers to operate under global awareness-consciousness. And the extended swarming chasers scenario, as follows, also shows in bold its interaction with the migrating global consciousness process.

hop_nodes $(\mathrm{C} 1, \mathrm{C} 2, \ldots \mathrm{Cm})$;

nodal $\left(\right.$ Area $=(X \min , X \max , Y \min , Y \max ) ; \mathrm{C}_{\mathrm{C}}$ number $=$ given $)$; T_number $=$ expected; T_destroyed $=0 ;$ Killed $=0$; Threshold $1=$ ..; Threshold $2=\ldots ;$ Threshold $3=\ldots ; \mathrm{R} 1=\ldots ; \mathrm{D} 1=\ldots ;$ Ratio $=\ldots$;

Start $=$ TIME); repeat

if (outside (WHERE, Area), move_random(Area)); if ( $T_{-}$ number $<=$ Threshold1, abort);

if (T_number / C_number >= Ratio, (move_outside(Area); abort));

If (TIME - Start $>$ Threshold2,

if (T_destroyed / T_number < Threshold3, abort));

Shift $=$ random $\left(\mathrm{dx}_{-} \mathrm{d}\right.$ y $)$; New $=$ WHERE + Shift;

if ((inside (New, Area); notseen(New, R1, chasers)), shift(Shift)); if(detect(targets, D1),

if(select_destroy(targets), increment(Killed)));

sleep(delay))

This extended chasers swarm scenario under the migrating global consciousness obtains important features which were absent in the initial traditional swarming functionality. First of all, it regularly gets the data on total area occupied by targets, instead of initial common area, into which its units should now move immediately. Second, it collects data on all targets destroyed individually and supplies it to the migrating consciousness which summarizes it from all chasers. Third, it may decide to terminate its operation if the total number of targets remains so small that there is no reason to continue the operation, or after a certain time interval the number of destroyed targets is so small in comparison to their total number that the whole operation for destroying targets proves to be unsuccessful. And fourth, if the number of discovered targets is overwhelmingly large in comparison with the number of available chasers, this may also abort the campaign as potentially dangerous for the whole chasers swarm. 


\section{Providing External Super-Consciousness}

We are showing here how to improve further and strengthen the overall consciousness of the chasers swarm. Despite organizing the internal consciousness of the swarm by allowing it constantly and randomly migrate between the chasers to reduce its vulnerability, we could not completely guarantee that the chaser holding the awareness focus cannot be accidentally destroyed by a target or just by own mechanical or software failure. To solve this problem, we may introduce a sort of external and global superconsciousness, which regularly tries to discover the presence of this wandering focus, which may be in any chaser and at any moment of time. And in case of its absence, can re- launch the internal migrating consciousness process from any chaser and continue overseeing its migration until another disappearance, with re-launching internal consciousness again, and so on. And all this can be organized continually and endlessly, as shown in Fig. 7 and by the corresponding SGL scenario that follows.

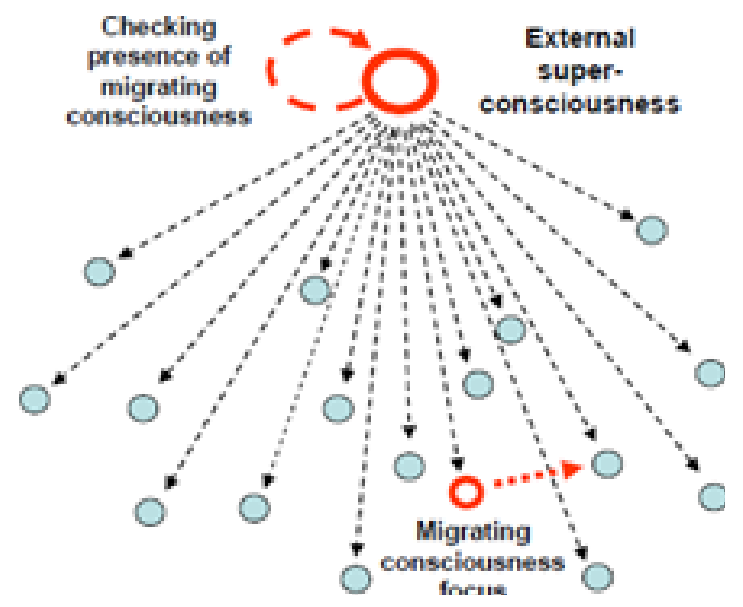

Figure 7: Providing external super-consciousness

Frontal (Chases_number, Targest_number, T a r g e t s _ a r e a , Targets_destroyed, Delay $=$...);

nodal (C_number, T_number, Area, T_destroyed, Conscious); repeat (

if(no(hop(all_chasers); Conscious == "active"), free (repeat (hop random(chasers); Conscious = "active";

stay (Chasers_number $=$ count $($ hop (all_chasers));

Targets_number $=$ count(hop(all_chasers); $\operatorname{radar}(\operatorname{targets}))$; Targets_area $=$ boundaries(hop(all_chasers); $\operatorname{radar}(\operatorname{targets})$ );

hop(all_chasers);

C number $=$ Chasers number;

$T$ number $=$ Targets number;

Area $=$ Targets_area;

T_destroyed $=$ Targets_destroyed);

sleep(Delay); Conscious $=$ nil)));

sleep(resume_time))

This external super-consciousness can reside outside the internally conscious swarm described before, within any other system, on any terrestrial or celestial distance from the chasers swarm, and even, despite absurdness and craziness of this idea, in a target itself, which can also migrate between the targets in their swarm. More super- conscious levels can be organized in SGL, each checking the presence of lower super-consciousness and re- launching it if needed, and which, in its turn, can be re-launched by the above standing super-consciousness if happens to disappear too.

SGL allows us to describe and implement fully distributed and parallel solutions for any problems, as shown in, including those formalizing main laws of the gestalt psychology and theory (like in [18]), and the above discussed external super-consciousness can be organized in a distributed manner too, fully residing within the chasers swarm, without any external intervention [15-19]. The overall consciousness of the swarm can always remain operational regardless of the varying number of interacting units in it, up to a single unit of its possible final reduction.

\section{Conclusions}

The paper investigated the possibility of using developed Spatial Grasp model and Technology (SGT) for simulating global awareness and consciousness in distributed dynamic systems. Despite the simplicity of the chosen example of enriching collectively operating swarm of randomly moving units with global awareness and migrating consciousness, it gives us hope for the potential use of SGT for simulation of much broader and complex areas linked with consciousness. SGT continues its development in different areas, including advanced mosaic-type operations in distributed systems, in providing philosophical and technological support of space conquest and advanced terrestrial and celestial missions, and others $[20,21]$. The initial idea of this paper was also accepted at THE SCIENCE OF CONSCIOUSNESS, TSC 2020 conference [22]. Current research interest also relates to investigation of possibility of modeling in SGL of fundamentals of quantum mechanics and space-time geometry, also connection between the brain's bimolecular processes and the basic structure of the universe [11]. For example, microtubule vibrations with links to consciousness [11] can be conceptually close to SGL, especially to its previous versions called WAVE, generating intelligent parallel waves covering and conquering distributed spaces $[15,16]$.

The latest SGL version can be quickly implemented even within standard university environments, similar to its previous versions in different countries under the author's supervision. The technology can be installed in numerous copies worldwide and deeply integrated with any other systems, actually acquiring unlimited power for simulation and management of the whole world, global awareness and consciousness including.

As the final words of this paper, the author wants to state that he did not pretend to clarify the general meaning of consciousness or make any formalization of it, but rather tried to show some practical higher-level system solutions which may intuitively relate to this mysterious word. And after studying a good deal of publications with diverse, opposing, often contradicting ideas on consciousness, he came to a conclusion that many of them, whatever fictitious and even ridiculous, can be productively used in creation of very practical systems. The latter may relate to intelligent management and control, industrial development, environmental protection, space research, security and defense -- all with the help of SGT based on half century of author's experience with large distributed networked systems and what is often called "artificial intelligence". 


\section{References}

1. C Koch (2018) What Is Consciousness? Scientific American. https://www.scientificamerican.com/article/what-isconsciousness/

2. AChella, R Manzotti (2011)Artificial consciousness, Research Gate. https://www.researchgate.net/publication/225838750_ Artificial_Consciousness

3. D Galland, M Grønning (2019) Spatial Consciousness, Research Gate. 1: 1-10. https://www.researchgate.net/ publication/330753755_Spatial_consciousness

4. M Massimini (2016) The Distribution of Consciousness: A Difficult Cartesian Chart, Research Gate 7: 3-15. https://www. researchgate.net/publication/307808562_The_Distribution_ of_Consciousness_A_Difficult_Cartesian_Chart

5. R Nelson, PA Bancel (2016) Exploring Global Consciousness, Research Gate https://www.researchgate.net/ publication/268001767_Exploring_Global_Consciousness

6. P Pavlidis (2015) "Social consciousness, education and transformative activity", Journal for Critical Education Policy Studies 13: 1-37. https://www.researchgate.net/ publication/325264094_Social_consciousness_education and_transformative_activity_Journal_for_Critical_ Education_Policy_Studies_Volume_13 $1 \overline{3}$ Number_2 October_20 $\overline{1} 5$

7. IM Evans (2015) The "Stream" of Consciousness: MindWandering, Introspection, Rumination, Meditation, and Mindfulness, in the book: How and Why Thoughts Change: Foundations of Cognitive Psychotherapy, Oxford University Press. https://www.oxfordclinicalpsych.com/view/10.1093/ med:psych/9780199380848.001.0001/med-9780199380848chapter-4

8. A Korala (2020) From visual perception, and towards a theory of consciousness. https://www.theory-of- consciousness.com/

9. CN Lazarus (2019) Does Consciousness Exist Outside of the Brain? Is consciousness actually a property of the universe like gravity or light?. https://www.psychologytoday.com/intl/ blog/think-well/201906/does-consciousness-exist- outsidethe-brain

10. G Cook (2020) Does Consciousness Pervade the Universe?, Scientific American. https://www.scientificamerican.com/ article/does-consciousness-pervade-the-universe/

11. S Hameroff, R Penrose (2014) Consciousness in the universe: a review of the 'Orch OR' theory, Physics of Life Reviews 11: 39-78. https://www.sciencedirect.com/science/article/pii/ S1571064513001188?via\%3Dihub

12. M Moran (2015) Network theory sheds new light on origins of consciousness, Medical Xpress. https://medicalxpress.com/ news/2015-03-network-theory-consciousness.html

13. P Ormen (2012) The qualities of pure consciousness. https:// prebenormen.com/personal-development/reflection/thequalities-of-pure-consciousness

14. PS Sapaty (1993) A Distributed Processing System, European Patent No. 0389655, Publ. 10.11.93, European Patent Office, Munich.

15. PS Sapaty (1999) Mobile Processing in Distributed and Open Environments, John Wiley \& Sons, New York.

16. PS Sapaty (2005) Ruling Distributed Dynamic Worlds, John Wiley \& Sons, New York.

17. PS Sapaty (2017) Managing Distributed Dynamic Systems with Spatial Grasp Technology, Springer.

18. PS Sapaty (2018) Holistic Analysis and Management of Distributed Social Systems, Springer.

19. PS Sapaty (2019) Complexity in International Security: A Holistic Spatial Approach, Emerald Publishing.

20. PS Sapaty (2019) Mosaic warfare: from philosophy to model to solutions, International Robotics \& Automation Journal 5: 157-166. https://medcraveonline.com/IRATJ/ IRATJ-05-00190.pdf

21. PS Sapaty (2020) Advanced terrestrial and celestial missions under spatial grasp technology, Aeronautics and Aerospace Open Access Journal 4: 81-88. https://medcraveonline.com/ AAOAJ/AAOAJ-04-00110.pdf

22. P Sapaty (2020) Symbiosis of Real and Simulated Worlds Under Global Awareness and Consciousness, abstract at: The Science of Consciousness Conference. https://eagle.sbs. arizona.edu/sc/report_poster_detail.php?abs $=3696$
Copyright: (C2020 Peter Simon Sapaty. This is an open-access article distributed under the terms of the Creative Commons Attribution License, which permits unrestricted use, distribution, and reproduction in any medium, provided the original author and source are credited. 